\title{
Quantification of D-dimer levels in
}

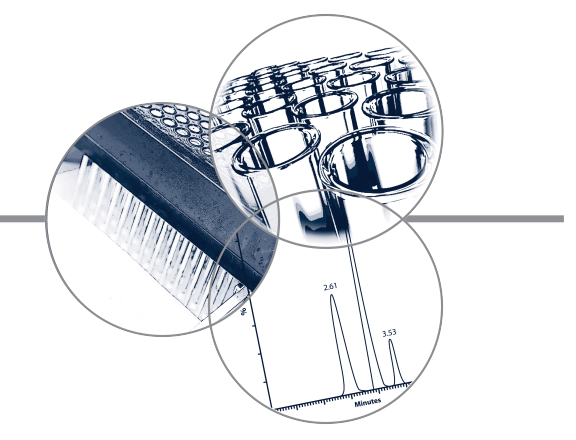
human saliva

Background: Plasma D-dimer tests are currently used to exclude deep vein thrombosis and pulmonary embolism. Human saliva has numerous advantages over blood as a diagnostic sample. The aims of our study were to develop a reliable immunoassay to detect $\mathrm{D}$-dimer levels in saliva, and to determine the correlation between salivary and blood D-dimer levels. Results/methodology: Saliva and blood samples were collected from 40 healthy volunteers. We developed a AlphaLISA ${ }^{\circledR}$ immunoassay with acceptable analytical performances to quantify D-dimer levels in the samples. The median salivary D-dimer levels were $138.1 \mathrm{ng} / \mathrm{ml}$ (morning) and $140.7 \mathrm{ng} / \mathrm{ml}$ (afternoon), and the plasma levels were $75.0 \mathrm{ng} / \mathrm{ml}$. Salivary D-dimer levels did not cor fe with plasma levels $(p=0.61)$. Conclusion: For the first time, we have quantified D-dimer levels in saliva there twofold higher $(p<0.05)$ than plasma levels. Further studies are required to demonstrate the clinical relevance/utility of salivary D-dimer in patients with confirmed deep vein thrombosis and/or pulmonary embolism.

Venous thromboembolism (VTE) is the third most common cause of death under the broad spectrum of cardiovascular diseases (CVD) [1]. VTE occurs when blood flow stagnates, especially when coupled with either a hypercoagulable state or vascular endothelial damage causing fibrin deposition, thereby trapping platelets, red blood cells and leukocytes to form venous thrombi. When such thrombi form in the deep veins such as in the thigh and calf, this is referred as deep venous thrombosis (DVT). Complications that may result from DVT include local venous insufficiency and leg ulceration, and if the thrombus breaks off, they can migrate to the lung (referred to as pulmonary embolism $[\mathrm{PE}]$ ), which is a life-threatening condition with over 30\% mortality. Plasma D-dimer test plays a critical role in clinical practice, particularly in DVT diagnosis and management since it rises more than 100-fold during acute DVT [2]. Combining it with a scoring system based on a clinical model, plasma D-dimer levels provide a high negative predictive value in the diagnosis of DVT [3] and PE [4]; hence, the need for diagnostic ultrasound imaging is reduced.

Fibrinogen is synthesized by the liver and secreted into the circulation [5]. In the presence of thrombin, fibrinogen can be cleaved into highly self-adhesive fibrin monomers [6]. Plasma D-dimer is a cross-linked fibrin degradation product. The presence of elevated levels of D-dimer in plasma is an indicator of thrombus formation. D-dimer is currently used to diagnose and manage a number of thrombosis-related clinical conditions, including disseminated intravascular coagulation, VTE [7,8], stroke, thrombolytic therapy [9] and PE [10]. However, there are many underlying conditions such as stroke, infection, pregnancy and heart failure that are unrelated to thrombus, but may lead to elevated D-dimer levels in the plasma resulting in a low positive predictive value [11-13]. In addition, plasma D-dimer levels are strongly and positively related to the occurrence of future venous thrombosis [2]. We therefore reasoned that there may be utility in exploring whether D-dimer can be detected in other body fluids that can be easily accessed and sampled in a non-invasive fashion. This could facilitate population screening to identify individuals at a high risk of developing thrombotic events. Indeed, Sivakumaran and Malton detected D-dimer levels in urine and raised the question of whether the ratio of plasma to urine levels could be more informative when screening asymptomatic populations to minimize the effect of inter-individual variability [14]. In our study, we explored whether D-dimer could be detected in saliva, and whether there is a correlation between plasma and saliva D-dimer levels taken from the same individuals.

Salivary secretions contain most of the biomolecules that are present in blood or urine [15-19]. Human saliva protein levels reflect our body's health and well-being, and about 20\% of proteins that are present in blood are also found in saliva [20], which highlights the diagnostic potential of saliva. Saliva does not clot
Xi Zhang', Yunxia Wan', Justin Cooper-White ${ }^{1,2}$, Goce Dimeski ${ }^{3,4}$, John Athertons ${ }^{5}$ \& Chamindie Punyadeera*1,2

'The Australian Institute for Bioengineering \& Nanotechnology, Australia

${ }^{2}$ The School of Chemical

Engineering \& the University of Queensland, Australia ${ }^{3}$ Chemical Pathology, Princes Alexandra Hospital, Brisbane, Australia

${ }^{4}$ School of Medicine, The University of Queensland, Brisbane,

Queensland, Australia

${ }^{5}$ Royal Brisbane and Women's

Hospital \& University of

Queensland, Australia

*Author for correspondence:

Tel.: +61734436953

Fax: +61 734436996

E-mail: c.punyadeera@uq.edu.au

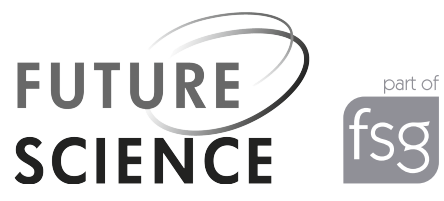




\section{Key Terms}

AlphaLISA ${ }^{\oplus}$ immunoassay:

PerkinElmer's bead-based chemiluminescent, no-wash assays which have high sensitivity, wide dynamic range and robust performance that compares advantageously with conventional ELISA.

D-dimer: A fibrin degradation product. A small protein fragment present in the blood after a blood clot is degraded by fibrinolysis.

LOD: LOD is the lowest quantity of a substance that can be distinguished from the absence of that substance (a blank value) in an assay.

Thrombin: A serine protease with a molecular weight of $36 \mathrm{kDa}$, it has multiple functions within the coagulation cascade, including converting fibrinogen to fibrin, factor XI to Xia, factor VIII to VIIla and factor V to Va.

Venous thromboembolism: Blood clot (thrombus) that forms within a vein. like blood, and its collection is minimally invasive $[17,19,21]$ Sa iva samples are relatively easy to collect and nandle by people with minimal training, plus it is easy to obtain multiple sample collections with a minimal risk for the collector of contracting blood-borne infectious organisms [22- Paliva has even been used as a biological

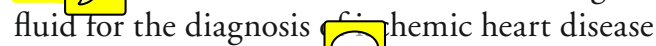
[15] and heart failure [25 2 letermine prognosis in oral cancers [26,27]. T $\Omega$ ms of our study were twofold: to develop a reliable immunoassay to detect D-dimer levels in saliva as a means of providing a preliminary reference range for this biomolecule in saliva and to determine whether the salivary D-dimer levels correlate with blood levels.

\section{Materials \& methods \\ - Participants}

This research was approved by the University of Queensland Medical Ethical Institutional Board and the Mater Hospital Medical Ethical Reviev Board. All participants were over 18 years of age and gave written informed consent before donating samples for our study. We recruited 40 healthy controls (20 males [19-36 years] and 20 females [19-25 years]) from the University of Queensland students and staff. The subjects were of European and Asian descent, were in good oral hygiene and had showed no symptoms of fever and/or respiratory tract infection. This information was provided to us via a sample questionnaire. Exclusion criteria obtained via a questionnaire included the existence of any comorbid oral disease (e.g., periodontal disease and gingivitis), autoimmune disease, active infection, malignancy, smoking, undergoing dental treatment, denture wearer, pregnancy and recent operation or trauma.

\section{- Samples}

Saliva and blood samples from each volunteer were collected during the same day. Blood samples were collected by an experienced phlebotomist (Queensland Medical Laboratory Pathology Services, University of Queensland Collection Centre, Australia) between 0900 and 1600 immediately after either the morning saliva collection or afternoon saliva collection. Blood samples were collected into sodium citrate tubes (Greiner VACUETTE ${ }^{\circledR}$ \# 454327, Greiner Bioone, Graz, Austria) and then immediately centrifuged at $500 \times \mathrm{g}$ at room temperature for $15 \mathrm{~min}$. The plasma samples were divided into aliquots, and stored at $-80^{\circ} \mathrm{C}$ until analyzed. Saliva samples were collected in sterile Falcon ${ }^{\mathrm{TM}}$ Polypropylene Conical Tubes $(50 \mathrm{ml}$, BD Bioscience, \#352070, CA, USA), divided into aliquots and stored at $-80^{\circ} \mathrm{C}$ until further analysis.

Salivary flow rates were calculated for all the study participants as described by Mahvash [28] in order to eliminate any confounding variables due to salivary gland dysfunction. Unstimulated resting saliva was collected by the drool meth $\rightarrow$ escribed by Navazesh and Christensen [29,30 subjects were asked to refrain from eating and drinking for $2 \mathrm{~h}$ prior to saliva collection in order to obtain a relatively constant baseline. We collected two saliva samples from each participant in the morning (0800-1200) and in the afternoon (1400-1600) to provide an indication of the diurnal variation of $\mathrm{D}$-dimer levels. The samples were collected and immediately aliquoted and de-identified. Prior to storage, saliva samples were centrifuged at $1500 \times \mathrm{g}$ for $15 \mathrm{~min}$ at $4^{\circ} \mathrm{C}$. The salivary supernatant as then diluted 40 times with AlphaLISA ${ }^{\circ}$ rmmunoassay Buffer (1X) and stored at $-80^{\circ} \mathrm{C}$ until analysis.

\section{- Salivary D-dimer AlphaLISA ${ }^{\circledR}$ immunoassay}

The D-dimer AlphaLISA kit (Product-No: AL290 C/F, Perkin Elmer, MA, USA) was used to determine the concentrations of $\mathrm{D}$-dimer in plasma and saliva samples. It contains a biotinylated anti-D-dimer monoclonal antibody, which binds to the streptavidin-coated donor beads, while the anti-D-dimer monoclonal antibody is conjugated to the acceptor beads. In the presence of D-dimer, the beads come into close proximity. After excitation at $680 \mathrm{~nm}$, donor beads will give out singlet oxygen molecules that trigger a cascade of energy transfer in the acceptor beads, resulting in a sharp peak of light emission at $615 \mathrm{~nm}$. AlphaLISA immunoassay uses monoclonal antibodies, and as such the specificity of this immunoassay is considerably high, with no cross-reaction with fibrinogen and $34 \%$ of crossreactivity with D-monomer (which is also a fibrin degradation product that can indicate fibrin formation) [31].

1 (5) ndards were usea to generate a standard curve. The commercially available D-dimer AlphaLISA kit is designed and validated for the measurement of human D-dimer levels in cirasma or cell culture medium. The kit . 2 t been validated for the use of measuring D-dimer level in human saliva samples. Saliva is a very complex, high viscoelastic biological 
fluid and as such it is important to minimize any influence that saliva might bring to the immunoassay (matrix effects).We have developed and validated an AlphaLISA immunoassay specific for the measurement of salivary D-dimer levels. For salivary measurements, the standards were prepared in pooled saliva $(n=10,2.5 \%$ of pooled saliva) and similarly when measuring the D-dimer levels in plasma, the standards were prepared in pooled plasma $(n=10,2.5 \%$ of pooled plasma) collected from healthy controls to eliminate any matrix effects. The samples were analyzed in 384 wells ProxiPlates ${ }^{\mathrm{TM}}$ in triplicat (D) rkin Elmer) with an exception of reducing the reaction volumes from 50 to $10 \mu \mathrm{l}$. In brief, the immunoassay consisted of $1 \mu$ of sample/analyte, $1 \mu \mathrm{l}$ of biotinylated antibody and acceptor bead mix (10 nM and $100 \mu \mathrm{g} / \mathrm{ml}$, respectively), and $8 \mu \mathrm{l}$ of streptavidin donor beads $(50 \mu \mathrm{g} / \mathrm{ml})$. The final concentration of acceptor beads, biotinylated antibody and streptavidin donor beads was $10 \mu \mathrm{g} / \mathrm{ml}, 1 \mathrm{nM}$ and $40 \mu \mathrm{g} / \mathrm{ml}$, respectively. We used an EnSpire ${ }^{\mathrm{TM}}$ plate reader (Perkin Elmer) to read the plates after $1.5 \mathrm{~h}$ of incubation at room temperature in the dark.

- Assay performance characteristics for the in-house modified salivary D-dimer

AlphaLISA

Recovery

To evaluate the suitability of AlphaLISA immunoassay for measuring salivary D-dimer levels, we spiked three commercial recombinant D-dimer (Product-No: D21012, Perkin Elmer MA, USA), measured the spiked pooled saliva samples using AlphaLISA immunoassay. An unspiked pooled saliva sample was measured at the same time. The percentage recovery of the three spiked saliva samples was calculated in reference to respective un-spiked pooled saliva samples in a single AlphaLISA immunoassay, using the following equation [32] $\bigcirc$

Insert equation

Intra- \& inter-assay coefficient of variation for the D-dimer AlphaLISA assay

To determine intra- and inter-assay variations, triplicates of saliva samples from 40 healthy controls were run in one AlphaLISA D-Dimer immunoassay and three independent AlphaLISA D-dimerimmunoassays, respectively [32].Intraand inter-assay variations were expressed by intra- or inter-assay $\% \mathrm{CV} . \% \mathrm{CV}$ was calculated using the following equation:

$\% \mathrm{CV}=($ Mean of SD $\times 100) /$ mean

LOD for the AlphaLISA immunoassay

To determine the LOD of the D-dimer immunoassay, 12 blanks (2.5\% pooled saliva diluted in 1X AlphaLISA Immunoassay buffer) were run in triplicate in one immunoassay run. The LOD for the salivary D-dimer immunoassay was read from a sigmoidal-dose response curve based on $\mathrm{LOD}$ signal counts derived from the equation [33]:

LOD signal count $=($ ave of blank signal count $)+3 \times \pm($ SD of blank signal $)$

\section{- Statistical analysis}

We used GraphPad Prism 5 software version 5.03 (GraphPad Software Inc., CA, USA) to perform all the statistical analyses. A D-dimer standard curve was generated by plotting the total raw AlphaLISA counts versus the concentration of D-dimer standards. A four-parameter logistic equation (sigmoidal dose-response curve with variable slope) coupled to a $1 / \mathrm{Y}^{2}$ data weighting were used to analyze the data.

We performed a Kolmogorov-Smirnov statistic in order to test for normal distribution of the clinical characteristics (continuous variables) of the volunteers before statistical analysis. Mann-Whitney U test and Wilcoxon test were performed on unpaired and paired data without normal distribution [34] Chi-square test for dichotomous variables to compare values from two groups. To investigate the relationship between salivary and plasma D-dimer levels, and between salivary flow rate in the morning and in the afternoon samples, we used nonparametric spearman product moment correlation coefficients.

\section{Results}

- Participants

In total 40 healthy volunteers were enrolled in the study between 22 August 2012 and 6 December 2012. The details of participants are shown in Table I.

\section{- Salivary flow rate measurements}

The salivary flow rates (median and interquartile range $[\mathrm{IQR}]$ ) for the study participants for morning and afternoon collections were $0.91 \mathrm{~g} / \mathrm{min}(0.470-1.257 \mathrm{~g} / \mathrm{min})$ and $1.1 \mathrm{~g} / \mathrm{min}$ (0.598-1.634 g/min), respectively (FIGURE I). There was a significant difference between 
Table 1. Characteristics of healthy controls.

\begin{tabular}{ll|}
\hline Parameter & Healthy controls $(\mathbf{n}=\mathbf{4 0})$ \\
Age (years) & $24(19-36)$ \\
Gender (M:F) & $20: 20$ \\
Ethnicity (Caucasian: Asian) & $19: 21$ \\
Body mass index & $22.7(18.0-29.2)$ \\
\hline
\end{tabular}

morning and afternoon salivary flow rates $(\mathrm{p}=0.0073)$, and there was a significant correlation between morning salivary flow rate to afternoon salivary flow rate $(\mathrm{p}<0.001$, correlation coefficient $\rho=0.760)$.

- Assay performance characteristics for the in-house modified salivary D-dimer AlphaLISA assay

The performance characteristic of the D-dimer immunoassay is summarized in TABLE 2. Intraand inter-assay $\mathrm{CV}_{s}$ for the salivary $\mathrm{D}$-dimer immunoassays were 5.7 and $7.4 \%$, respectively. The LOD values for the salivary and plasma D-dimer assays were 279 and $748 \mathrm{pg} / \mathrm{ml}$, respectively.

\section{- Salivary D-dimer concentrations in healthy controls}

Individual salivary D-dimer concentration is summarized in Figure 2. The D-dimer concentration in the saliva samples collected in the morning ranged from 6.0 to $1523.0 \mathrm{ng} / \mathrm{ml}$ with a median value of $138.1 \mathrm{ng} / \mathrm{ml}$ (IQR, 42.9 to $287.2 \mathrm{ng} / \mathrm{ml}$ ) (FIGURE 3).The D-dimer concentration in the saliva samples collected in the afternoon ranged from 11.2 to $2173.0 \mathrm{ng} / \mathrm{ml}$ with a median value of $140.7 \mathrm{ng} / \mathrm{ml}$ (IQR, 50.0 to $303.7 \mathrm{ng} / \mathrm{ml}$ ). There was no significant difference between D-dimer levels in the saliva samples collected in the morning and in the afternoon $(p=0.4907)$.

\section{- Plasma D-dimer concentrations in healthy controls}

The D-dimer concentration in the plasma samples ranged between 1.3 to $393.2 \mathrm{ng} / \mathrm{ml}$ with a median of $75.0 \mathrm{ng} / \mathrm{ml}$ (IQR, 42.8 to $176.7 \mathrm{ng} /$ $\mathrm{ml}$ ) (FIgure 3). There was a significant difference between morning saliva D-dimer levels and plasma D-dimer levels $(\mathrm{p}<0.05)$ as well as the afternoon's salivary D-dimer levels and plasma levels ( $\mathrm{p}<0.05)$ (FIGURE 3). However, by comparing individual samples using Wilcoxon matchedpairs signed rank test, we found no significant correlation between plasma and salivary D-dimer measurements taken from the same individual $(\mathrm{p}=0.605$ for morning saliva vs plasma and $\mathrm{p}=$ 0.955 for afternoon saliva vs plasma) (FIGURE 4).

\section{Discussion}

To our knowledge, this is the first time that the D-dimer levels in saliva has been investigated in healthy young adults. We observed that the D-dimer level in saliva was significantly higher than in the plasma from the same individuals and that there was no correlation between salivary and plasma D-dimer levels. Furthermore, salivary D-dimer levels did not significantly differ between morning and afternoon samples. The lack of correlation between the salivary and plasma $\mathrm{D}$-dimer levels may suggest that the D-dimer levels found in saliva could be produced in situ in the oral cavity. Saliva contains a thromboplastin-like substance and it has been identified as a tissue factor that is associated with
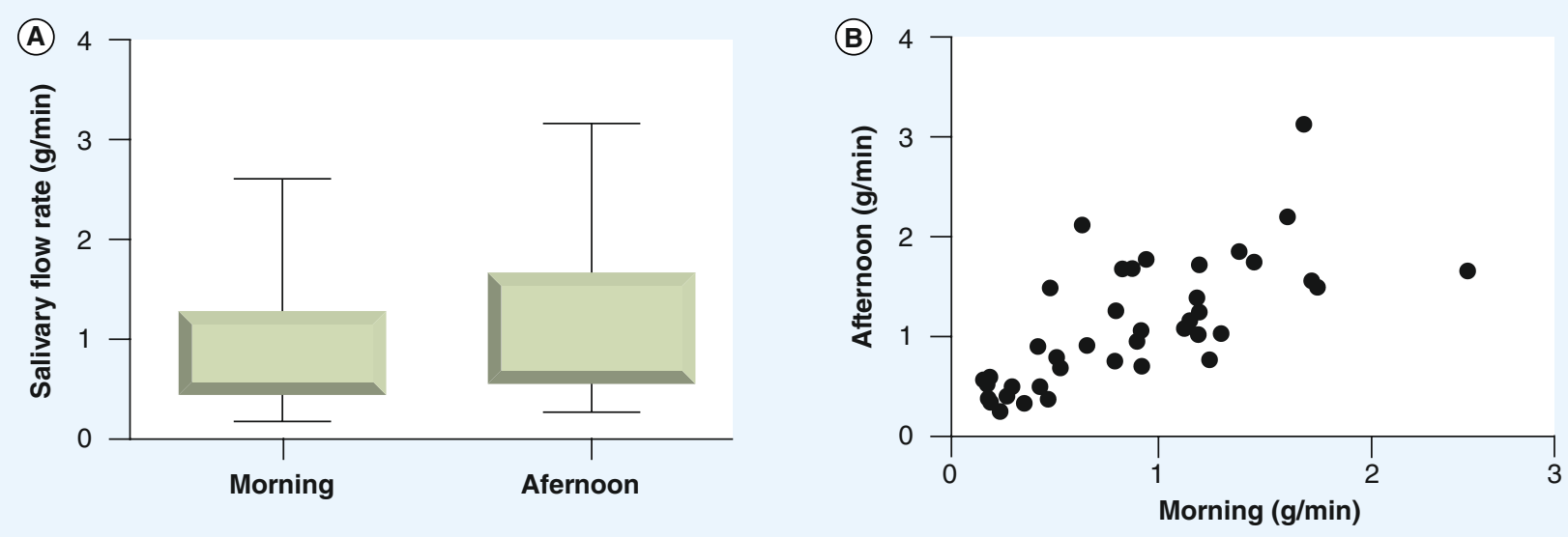

Figure 1. Comparison of salivary flow rates for samples collected in the morning and in the afternoon. (A) The Whisker plots for morning and afternoon salivary flow rates. (B) Correlations between morning and afternoon salivary flow rate (spearman correlation coefficient $\rho=0.760, p<0.001)$. 
Table 2. Performance characteristics of the in-house developed salivary D-dimer AlphaLISA ${ }^{\oplus}$ immunoassay.

\begin{tabular}{|c|c|c|c|c|}
\hline Analyte & $\%$ Recovery & $\begin{array}{l}\% \text { Intra-assay } \\
\text { variation } \\
\text { ( } \pm \text { std error) }\end{array}$ & $\begin{array}{l}\% \text { Inter-assay } \\
\text { variation } \\
\text { ( } \pm \text { std error) }\end{array}$ & LOD (pg/ml) \\
\hline D-dimer in saliva & 84.3 & $5.7(2.7)$ & $7.4(7.2)$ & 279 \\
\hline D-dimer in plasma & 85.5 & $6.7(4.3)$ & $7.5(3.1)$ & 748 \\
\hline
\end{tabular}

circulating cell-derived microparticles and these can become coagu [34]. Recently Berckmans et al. demonstrated that salivary tissue factor is associated with exosomes and microp $\mathbb{R}$ es that promote the clotting of blood [35]. study also showed that salivary tissue factor level was much higher than plasma tissue factor level ( 2000 vs $\sim 300 \mathrm{pg} / \mathrm{ml}$, respectively). Therefore, we hypothesize that the salivary tissue factor can promote blood clotting in capillaries in oral cavity, and to prevent these capillaries being blocked by the blood clot, fibrinolysis activities must also be present. Therefore, the $\mathrm{D}$-dimer production is increased in the capillaries surrounding the oral cavity leading to high D-dimer levels in saliva. However, more research is warranted to determine the exact mechanism leading to elevated salivary D-dimer levels and its role in saliva, and also to determine whether D-dimer levels differ between men and women.

In this study, we recruited 40 volunteers to collect their blood and saliva samples. These volunteers were all young healthy nonsmoking adults (ages $<35$ years) with no history of medical conditions, that is, lipemia. In addition, we also performed visual inspection of the citrate plasma samples and they showed no visible lipemia. Blood and saliva samples were generally collected at about the same time, which should provide a mirror image of pathological changes. In our study, since healthy people have functioning salivary gland functions, we collected only resting wouth whol samples from our study participants. Sarracan also be stimulated by eith d or mechanical stimulations which is ideal in a clinical setting when dealing with patients with impaired salivary gland functions. It will be interesting to find out whether the D-dimer levels are affected by stimulation.

In order to detect D-dimer in human saliva samples, we developed an existing commercially available immunoassay from Perkin Elmer (AlphaLISA D-dimer immunoassay). Due to the high concentrations of D-dimer in saliva, after diluting the samples 40 -times, the samples were still in the measuring range of the $\mathrm{D}$-dimer assay. In order to minimize the matrix effects, the standards for the assay needed to be prepared in the same matrix as the samples. We tried different dilutions of pooled healthy saliva as the diluent for the standards (data not shown), and 40 times diluted pool saliva was the best diluent to prepare the standards in and gave recoveries of $84.3 \%$.The performance characteristic of this assay indicated that the assay performance was reliable. The total incubation time for this

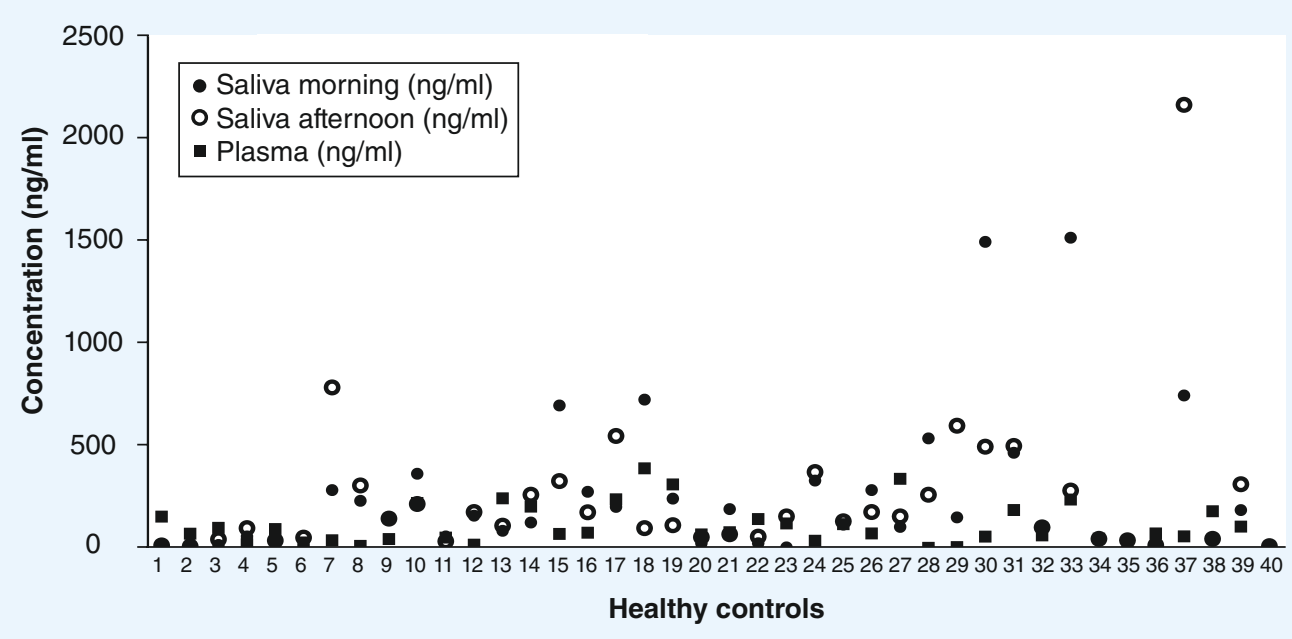

Figure 2. The D-dimer levels in saliva and in plasma of $\mathbf{4 0}$ healthy individuals. 


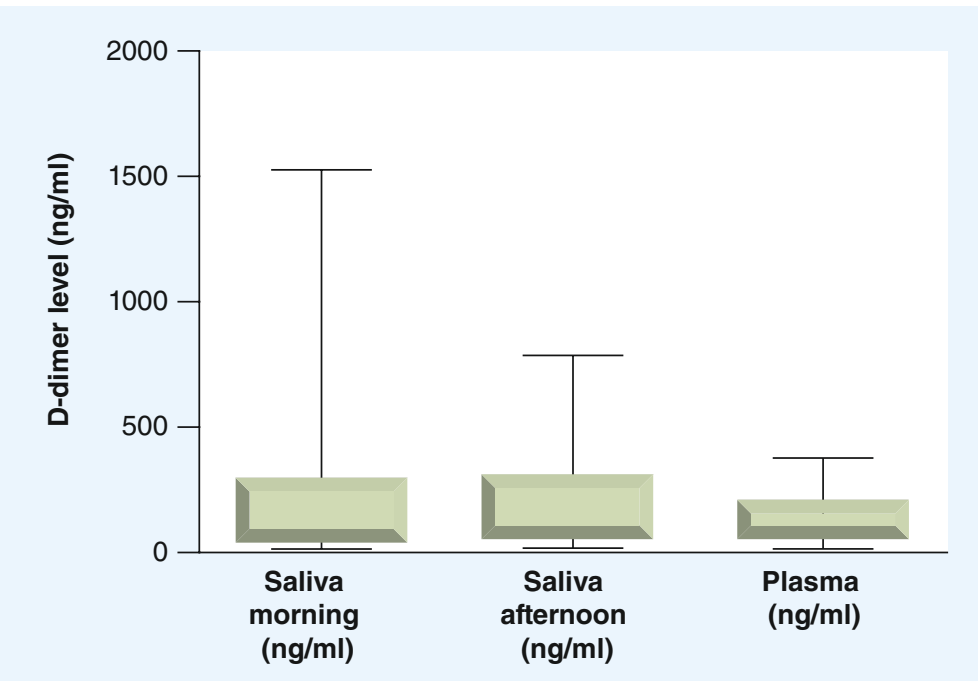

Figure 3. The D-dimer concentrations in saliva and plasma samples collected from healthy controls $(n=40)$.

immunoassay was $1.5 \mathrm{~h}$. The total time to perform D-dimer AlphaLISA assay for 40 samples (including samples preparation, reagents preparation and the addition of the samples onto the assay plate) would be around $3 \mathrm{~h}$. However, the assay time can be further reduced when one is implementing robotics automation and these assays can be run on the JANUS ${ }^{\circledR}$ automation work station. Furthermore, AlphaLISA technology is amenable for decentralized point-of-care testing and could either be placed at the doctor's office or could be used at a reference laboratory.

The medium level of salivary D-dimer was $150.9 \mathrm{ng} / \mathrm{ml}$ (IQR 56.9-87.2 $\mathrm{ng} / \mathrm{ml}$ ) and this information is likely to be useful when determining saliva reference ranges for D-dimer levels within a healthy population. In addition, there were no significant differences in the levels of D-dimer between morning and afternoon samples $(\mathrm{p}=0.5)$. Our findings are in line with Iversen $e t$ al. who showed that there was no D-dimer diurnal variation in plasma [37] (n) contrast, a study by Rudnicka et al. using large number of controls $(n=7667)$, found that the plasma D-dimer levels peaked at 14:00 h, but there was not a significant diurnal ion for plasma D-dimer level ( $\mathrm{p}=$ 0.17) [38] refore, a wide range of blood collection times should not contribute significantly to the inaccuracies of the results.

\section{Conclusion}

In summary, we have demonstrated that $\mathrm{D}$-dimer is detectable in saliva in a group of healthy individuals and that the levels are approximately twofold higher in saliva than in plasma. Furthermore, there was no significant correlation between salivary and plasma D-dimer levels. These findings suggest that salivary D-dimer may be produced in situ in the oral cavity. The potential for salivary $\mathrm{D}$-dimer testing needs to be explored once pre-analytical variables are optimized and standardized. Future studies should then investigate its clinical utility in both the diagnosis and screening for thromboembolic diseases. Measuring salivary D-dimer levels at an early stage of DVT and PE patients (without any treatment) could provide a reference range for salivary D-dimer level in thromboembolic diseases. By comparing the salivary D-dimer levels of healthy controls and DVT/PE patients, a cut-off value can be obtained for clinical diagnosis of these diseases.
(A)

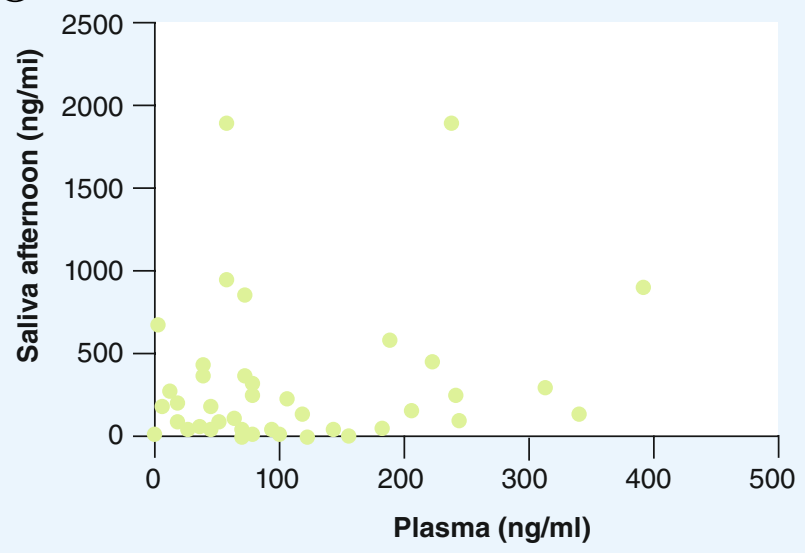

\section{(B)}

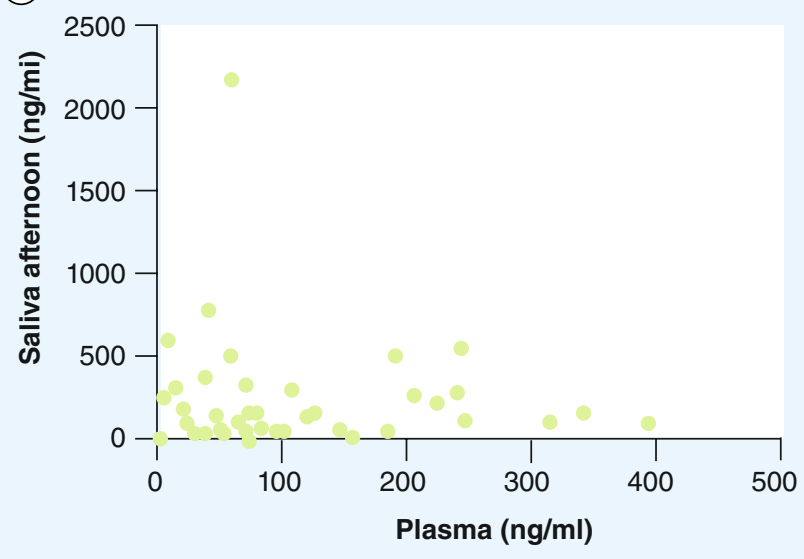

Figure 4. Comparison of salivary and plasma D-dimer levels. (A) D-dimer levels in the saliva collected from the morning to the plasma levels (spearman correlation coefficient $\rho=0.084, p=0.605$ ). (B) $D$-dimer level in the saliva collected from the afternoon to the plasma levels (correlation coefficient $\rho=-0.009, p=0.955$ ). 


\section{Future perspective}

CVD is becoming prevalent due to an aging and a growing population. Early diagnosis of CVD can greatly reduce morbidity and mortality. But most importantly early detection improves the chances of recovery for the patients, decreasing the burden on healthcare providers. Simple and cost-effective diagnostic fluids and methods are pivotal in reducing the growing burden of CVD. Saliva as a diagnostic/screening fluid has enormous potential over blood-based analysis. Data presented in this manuscript suggests that salivary D-dimer levels have great potential as a biomarker for the detection of DVT and PE. The long-term aim should be to develop a point-of-care testing device to facilitate large population based screening of DVT and PE. To date, we only have the salivary $\mathrm{D}$-dimer levels in 40 healthy controls, further and more comprehensive studies with sufficient number of VTE/PE patients may reveal its diagnostic utilty. This we plan to perform in the near future. In our study, the variations observed between individuals are larger in saliva than those observed in plasma, which may increase the number of false positives. Further and more comprehensive studies on confounding variables that may influence concentration changes may be warranted in the future.

Financial \& competing interests disclosure The authors would like to acknowledge financial support from the Queensland Government Smart Futures Fellowship Program (QGSFF), University of Queensland New Staff Research Funds (UQNSRSF 601252) and the University of Queensland Foundation Research Excellence Scheme. In addition, the authors wish to acknowledge clinical support from Queensland Medical Laboratory Pathology Services at the University of Queensland Health Centre. The authors have no other relevant affliations or financial involvement with any organization or entity with a financial interest in or financial conflict with the subject matter or materials discussed in the manuscript apart from those disclosed.

No writing assistance was utilized in the production of this manuscript.

\section{Ethical conduct of research}

The authors state that they have obtained appropriate institutional review board approval or have followed the principles outlined in the Declaration of Helsinki for all human or animal experimental investigations. In addition, for investigations involving human subjects, informed consent has been obtained from the participants involved.

\section{Executive summary}

\section{Assay performance characteristics for the in house developed salivary D-dimer AlphaLISA ${ }^{\circledR}$ assay}

- We used AlphaLISA sandwich immunoassay technology to detec nimer levels in saliva. We accounted for immunoassay matrix effects by using a $2.5 \%$ pooled saliva to prepare standard curve 2 termine D-dimer concentrations. Intra- and inter-assay CVs for the salivary D-dimer immunoassays were at acceptable level (5.7 and $7.4 \%$, respectively).

\section{Salivary \& plasma D-dimer concentrations in healthy controls}

- In a healthy population $(\mathrm{n}=40)$, the salivary D-dimer level ranged from 6.0 to $1523.0 \mathrm{ng} / \mathrm{ml}$ with a median value of $138.1 \mathrm{ng} / \mathrm{ml}$, which was approximately twofold higher than in plasma (ranged between 1.3 and $393.2 \mathrm{ng} / \mathrm{ml}$ with a median of $75.0 \mathrm{ng} / \mathrm{ml}$ )

\section{Future perspective}

- Further studies are required to understand the biological role of D-dimers in saliva.

\section{References}

Papers of special note have been highlighted as: - of interest

-. of considerable interest

1 Goldhaber SZ, Bounameaux H. Pulmonary embolism and deep vein thrombosis. Lancet 379(9828), 1835-1846 (2012).

2 Cushman M, Folsom AR, Wang L et al. Fibrin fragment D-dimer and the risk of future venous thrombosis. Blood 101(4), 1243-1248 (2003).

3 Wells PS, Anderson DR, Rodger M et al. Evaluation of D-dimer in the diagnosis of suspected deep-vein thrombosis. N. Engl. J. Med. 349(13), 1227-1235 (2003).
- Clinical application of D-dimer test.

4 Rathbun SW, Whitsett TL, Vesely SK, Raskob GE. Clinical utility of D-dimer in patients with suspected pulmonary embolism and nondiagnostic lung scans or negative CT findings. Chest 125(3), 851-855 (2004).

5 Ritchie DG, Levy BA, Adams MA, Fuller GM. Regulation of fibrinogen synthesis by plasmin-derived fragments of fibrinogen and fibrin: an indirect feedback pathway. Proc. Natl Acad. Sci. USA 79(5), 1530-1534 (1982).

6 Mosesson MW. Fibrinogen and fibrin structure and functions. J. Thromb. Haemost. 3(8), 1894-1904 (2005).
7 Brill-Edwards P, Lee A. D-dimer testing in the diagnosis of acute venous thromboembolism. Thromb. Haemost. 82(2), 688-694 (1999).

8 Wells PS, Anderson DR, Rodger M et al. Evaluation of D-dimer in the diagnosis of suspected deep-vein thrombosis. N. Engl. J. Med. 349(13), 1227-1235 (2003).

9 Tripodi A. D-dimer testing in laboratory practice. Clin. Chem. 57(9), 1256-1262 (2011).

10 Froehling DA, Daniels PR, Swensen SJ et al. Evaluation of a quantitative D-dimer latex immunoassay for acute pulmonary embolism diagnosed by computed tomographic 
angiography. Mayo Clin. Proc. 82(5), 556-560 (2007).

11 Adam SS, Key NS, Greenberg CS. D-dimer antigen: current concepts and future prospects. Blood 113(13), 2878-2887 (2009).

12 Dirix LY, Salgado R, Weytjens R et al. Plasma fibrin D-dimer levels correlate with tumour volume, progression rate and survival in patients with metastatic breast cancer. $\mathrm{Br} \mathrm{J}$. Cancer 86(3), 389-395 (2002).

13 Brotman DJ, Segal JB, Jani JT, Petty BG, Kickler TS. Limitations of D-dimer testing in unselected inpatients with suspected venous thromboembolism. Am. J. Med. 114(4), 276282 (2003)

14 Sivakumaran M, Malton N. Plasma D-dimer measurement as a predictor of venous thrombosis. Blood 102(13), 4618-4619 (2003).

15 Punyadeera C, Dimeski G, Kostner K, Beyerlein P, Cooper-White J. One-step homogeneous $\mathrm{C}$-reactive protein assay for saliva. J. Immunol. Methods 373(1-2), 19-25 (2011).

16 Schulz BL, Cooper-White J, Punyadeera CK. Saliva proteome research: current status and future outlook. Crit. Rev. Biotechnol. (2012).

17 Mohammed R, Leigh Cambell J, CooperWhite J, Dimesky G, Punyadeera C. The impact of saliva collection and processing methods on Crp, Ige, and myoglobin immunoassays. Clin. Transl. Med. 1(1), 19 (2012).

18 Pfaffe T, Cooper-White J, Beyerlein P, Kostner K, Punyadeera C. Diagnostic potential of saliva: current state and future applications. Clin. Chem. 57(5), 675-687 (2011).

19 Topkas E, Keith P, Dimeski G, Cooper-White J, Punyadeera C. Evaluation of saliva collection devices for the analysis of proteins. Clin. Chim. Acta 413(13-14), 1066-1070 (2012).
20 Hu S, Li Y, Wang J et al. Human saliva proteome and transcriptome. J. Dent. Res. 85(12), 1129-1133 (2006).

21 Punyadeera C, Dimeski G, Kostner K, Beyerlein P, Cooper-White J. One-step homogeneous $\mathrm{C}$-reactive protein assay for saliva. J. Immunol. Methods 373(1-2), 19-25 (2011)

22 Kiyosawa K, Sodeyama T, Tanaka E et al. Hepatitis $\mathrm{C}$ in hospital employees with needlestick injuries. Ann. Intern Med. 115(5), 367-369 (1991).

23 Oksenhendler E, Harzic M, Le Roux JM, Rabian C, Clauvel JP. HIV Infection with seroconversion after a superficial needlestick injury to the finger. N. Engl. J. Med. 315(9), 582-582 (1986).

24 Pfaffe T, Cooper-White J, Beyerlein P, Kostner K, Punyadeera C. Diagnostic potential of saliva: current state and future applications. Clin. Chem. 57(5), 675-687 (2011).

- Described the potential of salivary diagnostic test in detail.

25 Foo JY, Wan Y, Kostner K et al. NT-ProBNP levels in saliva and its clinical relevance to heart failure. PLoS ONE 7(10), e 48452 (2012).

26 Ovchinnikov DA, Cooper MA, Pandit P et al. Tumor-suppressor gene promoter hypermethylation in saliva of head and neck cancer patients. Transl. Oncol. 5(5), 321-326 (2012).

27 Nagadia R, Pandit P, Coman WB, CooperWhite J, Punyadeera C. miRNAs in head and neck cancer revisited. Cell. Oncol. (Dordr.) 36(1), 1-7 (2013).

28 Navazesh M, Kumar SK, University of Southern California School of Dentistry. Measuring salivary flow: challenges and opportunities. J. Am. Dent. Assoc. (1939)139 (Suppl.), 35S-40S (2008).
29 Navazesh M. Methods for collecting saliva. Ann. NYAcad. Sci. 694, 72-77 (1993).

30 Poll EM, Kreitschmann-Andermahr I, Langejuergen Y et al. Saliva collection method affects predictability of serum cortisol. Clin. Chim. Acta 382(1-2), 15-19 (2007).

31 Perkin Elmer. Human D-dimer kit technical data sheet. AlphaLISA ${ }^{\circledR}$ Research Reagents TDS-AL290-01, (2011)

32 Jaedicke KM, Taylor JJ, Preshaw PM. Validation and quality control of ELISAs for the use with human saliva samples. $J$. Immunol. Methods 377(1-2), 62-65 (2012).

33 Armbruster DA, Pry T. Limit of blank, limit of detection and limit of quantitation. Clin. Biochem. Rev. 29(Suppl. 1), S49-S52 (2008).

34 Hart A. Mann-Whitney test is not just a test of medians: differences in spread can be important. BMJ 323(7309), 391-393 (2001).

35 Furie B, Furie BC. Mechanisms of thrombus formation. N. Engl. J. Med. 359(9), 938-949 (2008).

36 Berckmans RJ, Sturk A, Van Tienen LM, Schaap MC, Nieuwland R. Cell-derived vesicles exposing coagulant tissue factor in saliva. Blood 117(11), 3172-3180 (2011).

- Provided evidence that blood coagulation factors are presented in saliva.

37 Iversen PO, Groot PD, Hjeltnes $\mathrm{N}$, Andersen TO, Mowinckel MC, Sandset PM. Impaired circadian variations of haemostatic and fibrinolytic parameters in tetraplegia. $\mathrm{Br}$. J. Haematol. 119(4), 1011-1016 (2002).

38 Rudnicka AR, Rumley A, Lowe GDO, Strachan DP. Diurnal, seasonal, and blood-processing patterns in levels of circulating fibrinogen, fibrin D-Dimer, C-reactive protein, tissue plasminogen activator, and von Willebrand factor in a 45-year-old population. Circulation 115(8), 996-1003 (2007). 
